


\section{El equilibrio personal y profesional como valor de la cultura corporativa}

Amaia Arribas Urrutia

- Doctora y licenciada en Ciencias de la Información por la Universidad del País Vasco, España

- Profesora investigadora en Comunicación en el Tecnológico de Monterrey, Campus Estado de México

- Su labor profesional se ha desarrollado como periodista en varios medios de radio y prensa escrita, documentalista en una institución pública, copy en una agencia de publicidad, y docente en el ámbito universitario y fuera de él (formación tecnológica a empresas)

- Como investigadora en Comunicación Empresarial, ha participado con ponencias y comunicaciones en diversos foros de Comunicación Social

- Es miembro del Sistema Nacional de Investigadores de México

- Autora de artículos especializados en publicaciones nacionales e internacionales

- amaya.arribas@itesm.mx 
Resumo

As organizações compreenderam a necessidade de conciliar vida pessoal e profissional para o aumento da produtividade e para proporcionar mais satisfação a seus funcionários. O sucesso de uma organização é baseado na flexibilidade, que vem a ser um traço da identidade cultural de empresas que buscam se adaptar a diversos perfis humanos. Este texto apresenta uma política de implantação da flexibilidade e demonstra que as empresas cuidam cada vez mais de seus funcionários a fim de retê-los, já que a mobilidade no mercado de trabalho permite que se opte por organizações que forneçam condições mais satisfatórias.

PALAVRAS-CHAVE: RESPONSABILIDADE SOCIAL • FAMÍLIA • FLEXIBILIDADE • TRABALHO

\section{Abstract}

Organizations have come to understand the need to combine personal life with work, in order to increase productivity and provide more satisfaction for their employees. The organization's success is based on flexibility, which represents a sign of the cultural identity of organizations that seek to be adapted to varied human profiles. This text presents a policy for implementation of the flexibility, and shows that companies are more and more caring for their employees in order to retain their services, inasmuch as labor market mobility allows them to work for organizations that offer conditions that are more satisfactory.

KEYWORDS: SOCIAL RESPONSIBILITY • FAMILY • FLEXIBILITY • WORK

\section{Resumen}

Las organizaciones han entendido la necesidad de conjugar la vida personal con la profesional para lograr mayores grados de productividad y satisfacción de los empleados. El éxito de una organización se asienta en la flexibilidad y ésta llega a ser una de las señas de identidad de la cultura de muchas empresas que buscan adaptarse a perfiles diversos. Se presenta una política de implantación de la flexibilidad y se demuestra que las empresas cuidan cada vez más de sus empleados para retenerlos, ya que la movilidad del mercado laboral les permite trabajar en aquellas organizaciones cuyas condiciones les resulten más satisfactorias.

PALABRAS CLAVE: RESPONSABILIDAD SOCIAL • FAMILIA • FLEXIBILIDAD - TRABAJO 

a satisfacción en el trabajo está basada en ocho pilares: ambiente laboral motivador; momentos de celebración; equilibrio; ambiente abierto y franqueza; aprobación y valoración; sueldo y evaluación; oportunidades y retos; y grados de libertad (factores de medición que emplea la empresa BetterBeCompany para determinar cómo los empleados afrontan su trabajo y cuánta satisfacción obtienen). Ahora hay que sumarle uno más: el Work Life Balance (WLB) (MACINNES, 2005, p. 41). Ha nacido un nuevo concepto en la terminología de la gestión empresarial. Literalmente se trata del balance entre la vida profesional y familiar, o lo que algunos autores prefieren llamar "empresa familiarmente responsable".

Este concepto se opone a aquella creencia tradicional sostenida por algunas teorías organizacionales (Teoría de la Burocracia, Teoría de la Dirección Científica y Teoría Clásica), según las cuales para alcanzar el éxito profesional era necesario dejar en segundo plano la vida personal. Desde estas perspectivas, la conciliación de la vida personal y laboral, o la denominada flexibilidad laboral, era vista como un beneficio social ventajoso tan sólo para determinados trabajadores. Sin embargo, hoy en día está extendida la creencia de que este equilibrio favorece la motivación y el compromiso. Por lo tanto, también la eficiencia en el puesto de trabajo.

El incentivo económico no es siempre el más deseado por muchos de los empleados. Muchos de ellos prefieren ganar menos y tener más tiempo libre. Además, la idea de que un grado de responsabilidad profesional elevado implique estar disponible las 24 horas del día está cada vez más desterrada, pues el compromiso con la empresa se mide con otros indicadores.

Se debe explotar el concepto de que si el trabajador se siente satisfecho en su trabajo incrementa el margen de beneficios. A pesar de que el camino para conseguir el equilibrio entre trabajo y vida personal será largo, a la empresa le interesa fomentarlo, ya que ésta será su ventaja competitiva para atraer el talento de los mejores profesionales. De este modo, la empresa conseguirá generar compromiso, lo que conforma uno de los principales objetivos de los directivos de recursos humanos.

Ya existen estudios (por ejemplo, el realizado por la empresa española reclutadora de empleo Monster.es) que reflejan que los mejores empleados son aquéllos capaces de mantener un buen equilibrio entre sus responsabilidades profesionales y personales. Además, es posible ofrecer flexibilidad laboral sin perder eficacia, algo aconsejable si tenemos en cuenta que el fenómeno de la adicción al trabajo es cada vez más frecuente entre altos cargos. 
Mucho se ha dicho y escrito sobre la importancia de la cultura organizativa como elemento diferenciador de las empresas que obtienen mejores resultados en su sector. Este nuevo valor en la cultura corporativa es determinante para la atracción los candidatos o la fuga de talentos.

\section{La orientación de la empresa}

La dinámica cultural, la ideología corporativa y la orientación estratégica son las variables culturales que definen la personalidad de la empresa. La orientación estratégica está constituida por las prioridades que establece la empresa para cumplir sus metas corporativas. No se trata de una declaración única de intenciones, sino que compromete a la empresa en el plano operativo.

Cada orientación estratégica se caracteriza por una suma de valores culturales que la definen. Las más comunes son la orientación estratégica a la empresa; al cliente; al empleado; a los resultados; a los costos; a la innovación; y a la tecnología.

Cuando una organización decide orientarse a la empresa, se está concentrando en el poder, y ofrece pocas posibilidades de flexibilidad a sus trabajadores. Esto repercute enormemente en la posibilidad de conseguir un buen equilibrio entre la vida personal y profesional. En la mayoría de estas empresas, cuya dirección de recursos humanos recae en hombres, manifiesta que la mayoría de los trabajadores estaría dispuesta a ganar menos dinero para disponer de más tiempo libre. Sin embargo, asegura que una demostración de alto compromiso con la empresa implica trabajar muchas horas. En este tipo de organizaciones la cultura del poder es muy difícil de cambiar y es claramente condicionante de los modelos empresariales, principalmente de países latinos.

Las organizaciones más amigables con el trabajador responden a una cultura orientada a la persona, es decir, al empleado. Los recursos humanos son considerados el principal activo de la empresa y, por ello, ofrecen las máximas condiciones de flexibilidad a sus trabajadores (teletrabajo, periodos sabáticos, flexibilidad horaria...). Se fomenta esta flexibilidad porque existe una gran confianza en la calidad del empleado. Se fomenta la transparencia en promociones y ascensos, con amplias prestaciones sociales, lo cual promueve el sentido de pertenencia.

En este tipo de orientación, los directivos, en su mayoría mujeres y de países del norte de Europa y Estados Unidos (ÁLVAREZ, 2006), manifiestan su desacuerdo en que un buen alto cargo deba estar disponible para trabajar a cualquier hora del día. Tampoco aceptan esa creencia con relación a la mayoría de los empleados.

Hay un tercer tipo de empresas que se sitúa en una posición intermedia, ya que ofrece cierta flexibilidad siempre que no perjudique el buen rendimiento del trabajador. 
Se trata de la orientación a los resultados, donde se pone especial énfasis en la planificación, control, y definición de objetivos; en un estricto cumplimiento de los plazos de ejecución de las tareas, y a la vez una alta flexibilidad funcional y orgánica; gran movilidad interna; buena disposición personal para la resolución de imprevistos, y una retribución variable en función de los resultados.

Normalmente son grandes multinacionales de países centroeuropeos (ÁLVAREZ, 2006) las que ofrecen algunas condiciones clásicas de flexibilidad laboral, como horario a tiempo parcial y posibilidad de elegir el período de vacaciones. Este modelo de empresa manifiesta su desacuerdo con la premisa de que los empleados anteponen cada vez más su vida profesional a la personal, y contraria la creencia de que la mejor forma de retener a los empleados es ofrecerles incentivos económicos.

\section{La flexibilidad como elemento competitivo}

Se entienden por elementos configuradores de la flexibilidad la posibilidad de disponer de horario a tiempo parcial; períodos sabáticos; bajas más extensas de lo estipulado por ley; posibilidad de poder trabajar desde casa; libertad para escoger el período de vacaciones, y la opción de compartir el puesto de trabajo o incluso intercambiarlo con otro compañero (ÁLVAREZ, 2006).

Implantar la flexibilidad en un modelo organizativo implica profundas transformaciones en la dinámica de las empresas. Si partimos del contexto de que las organizaciones deben de ser capaces de responder en cualquier momento a los requerimientos de los clientes, entonces la flexibilidad se convierte en un elemento que determina la capacidad competitiva de las mismas. Pero, a la vez, las organizaciones tienen la responsabilidad de facilitar los métodos para que los empleados puedan compatibilizar sus obligaciones profesionales con su vida privada ${ }^{1}$.

La flexibilidad es una política de doble sentido. Es un arma para que las empresas ganen en efectividad y eficacia, siendo un instrumento básico para que la carga de trabajo no se convierta en obstáculo insuperable del desarrollo individual, personal y familiar. Así, la flexibilidad se convierte en una ventaja competitiva al transformar la empresa en un punto de atracción y retención del talento ${ }^{2}$.

1 Las opciones de flexibilidad no son un derecho, es decir, la empresa debe ayudar al empleado a gestionar sus responsabilidades tanto profesionales como personales, pero cada caso debe tratarse de manera individualizada, y en función de ese análisis se aprobará o rechazará las propuestas de flexibilidad de cada empleado.

2 Estudios realizados en Estados Unidos muestran que la calidad del puesto de trabajo (esto incluye la flexibilidad) son mejores métodos para mejorar la productividad, la satisfacción en el trabajo y el compromiso personal que el salario y los beneficios. 
Se trata de una flexibilidad en sentido global, ya que estudios internos destacan las preocupaciones de los empleados por encontrar el equilibrio entre el trabajo y la vida privada. Además se agudiza cada vez más la tendencia a que los beneficios de un programa de flexibilidad sean comunes a hombres y mujeres.

Lo que persiguen las empresas es el compromiso, no la permanencia, y este compromiso se consigue ofreciendo una mayor flexibilidad. Esto es, la presencia no es lo mismo que el rendimiento. La valoración de un empleado depende de su rendimiento y del valor que aporte a la organización, no de las horas pasadas en la oficina. La cultura de empresa debe basarse en la confianza en la persona, no en el control.

Podemos justificar la flexibilidad porque es una necesidad, ya que si se logra equilibrar la vida personal y laboral se facilita que los empleados puedan contribuir con toda su energía y talento a los objetivos de su empresa. También hay que aclarar que igualdad no significa tratar a todas las personas por igual, sino reconocer las diferencias para que todos los empleados tengan las mismas oportunidades de realizar aportaciones y tener éxito.

Es bueno emplear la flexibilidad, pero también es positivo no usarla cuando hay que otorgar prioridad a los compromisos con los clientes. Asimismo, estas opciones de flexibilidad tienen que ser lo suficientemente modificables como para poder alterarlas cuando las circunstancias personales y profesionales cambien.

Por último, la flexibilidad no perjudica el desarrollo de la carrera personal, ya que no se penaliza al empleado por utilizarla. Las opciones de flexibilidad tienen un impacto positivo sobre la productividad, la moral, la capacidad de concentración y el compromiso con la empresa. La responsabilidad en el trabajo, el rendimiento y las capacidades de cada uno continúan siendo los criterios básicos para la promoción. Aunque también hay que ser conscientes de que no todas las oportunidades que se presentan a lo largo de la carrera profesional son compatibles con las opciones de flexibilidad.

\section{Método de implantación de una política de flexibilidad}

El uso de las nuevas tecnologías en la organización está transformando la cultura empresarial, así como los profundos cambios organizativos están potenciando la utilización intensiva de dichas tecnologías, sobre todo las asociadas a Internet. Todo ello se traduce principalmente en dos tendencias: asistimos a un cambio en el marco de las relaciones entre la empresa y los empleados y vemos cómo se superan las barreras físicas derivadas de la obligatoriedad de acudir al centro de trabajo (teletrabajo).

La relación antes era de jefe-subordinado (todavía en muchas organizaciones sigue siendo así), e incluso se producía un comportamiento paternalista donde el director acos- 
tumbraba a seguir al empleado para controlarlo y decirle cómo tenía que hacer las cosas. En la actualidad se constata la reducción del número de niveles jerárquicos, puesto que éstos existen porque el número de personas que un superior puede controlar con eficacia es limitado. Con esta reducción se diminuye el coste de funcionamiento de la estructura; se facilita la coordinación entre niveles; se mejoran las comunicaciones, y se perfeccionan las tareas de planificación y control. Ahora, en las organizaciones modernas, caracterizadas por la horizontalidad ${ }^{3}$, muchos empleados trabajan lejos de sus jefes, por lo que es imposible la relación de antes. Los trabajadores asumen nuevas cuotas de responsabilidad individual y un alto grado de iniciativa personal.

Este cambio de cultura en la empresa, donde cada uno es responsable de su propio trabajo, es lo que facilita la eliminación de barreras de espacio y tiempo en la realización de tareas.

Se consigue fomentar la flexibilidad con el teletrabajo, mediante el cual se mejora la productividad y se incrementa la satisfacción de los empleados, que pueden gestionar sus tiempos de manera más eficaz y equilibrar su vida profesional y personal. Ello implica una cultura de confianza y un sistema de evaluación basado en los resultados y no en las horas de permanencia en una oficina, reduciendo además los tiempos muertos (desplazamientos, dependencias de clientes...).

Ya no cabe hablar de centralización dentro de la compañía, sino que ésta saca fuera de sus paredes sus tentáculos para organizarse. Por ello, durante estos últimos años, el teletrabajo está despertando un enorme interés en las sociedades desarrolladas. El fuerte apoyo que recibe por parte de las administraciones y la gran cantidad de iniciativas de promoción puestas en marcha lo convierten en un tema de gran actualidad, favoreciendo el desarrollo de diversas experiencias.

Como afirma el profesor García Jiménez (1998), no cambia la naturaleza de la tarea, sino el escenario y el tipo de relación. El teletrabajo utiliza como materia prima la información; como herramienta, el ordenador, y, como vehículo, las telecomunicaciones.

La adopción del teletrabajo por parte de la empresa requiere cambios en la organización y en las políticas comunicacionales de nuevos entornos, como son la estructura en red y una política de redimensión y de nuevos entornos. El acceso de las empresas

3 Tom Peters, gurú del management, postulaba ya en su libro En busca de la excelencia, en coautoría con Robert Waterman (1984) la necesidad de flexibilizar las estructuras organizativas. Algunas empresas, como Astra, del grupo Merck, han optado por estructurarse como organizaciones horizontales desde su inicio. En vez de organizarse alrededor de áreas funcionales, se han estructurado en procesos de negocios orientados al mercado, desde el desarrollo del producto hasta su almacenamiento y distribución. 
a Internet está desarrollando la iniciativa de crear redes digitales que van a agrupar a trabajadores y empresas virtuales.

Además de las condiciones técnicas, tienen que adoptarse determinadas medidas organizativas para el desarrollo del teletrabajo o para la creación del mismo. La integración del teletrabajo en la organización conlleva numerosos problemas. Por ejemplo, ¿cuál debe ser el contenido del trabajo? Me refiero a cuáles son las partes de las tareas a realizar en forma de teletrabajo. Un aspecto importante es la coordinación, es decir, cómo se logra la integración del teletrabajo en los procesos empresariales de forma eficiente. Y por último, el tema de la cooperación, es decir, cómo tiene que organizarse la colaboración con los colegas en la empresa o con los que realizan teletrabajo.

Esta modalidad reduce el nivel de absentismo y dificulta la unión y contacto entre los trabajadores, lo que puede incidir en un menor índice de conflictividad laboral (ARRIBAS, 2002). También permite una mayor laxitud en el establecimiento del marco de gestión empresarial. La no especificación de las obligaciones en los contratos permite y a menudo estimula la flexibilidad a la vez que evita la inhibición en el flujo de información motivada por los contratos clásicos en los que aquélla se retiene por temor a ser utilizada en contra del informador.

El teletrabajo produce la posibilidad de incrementar las retribuciones percibidas como consecuencia del aumento de productividad, e incluso a través de la prestación de servicios a distintas empresas adoptando la figura de teletrabajador independiente. Por tanto, atenúa las graves dificultades del desempleo y ofrece a quienes lo practican mayores facilidades para trabajar en régimen de autoempleo. Además, la formación recibida y los conocimientos adquiridos sobre informática y telecomunicaciones otorgan al trabajador una mejor posición en el mercado de trabajo.

La comunicación interna se ve seriamente afectada con la implantación del teletrabajo. En el caso de organizaciones que incorporen programas de teletrabajo, la comunicación interna se debe incrementar con el objetivo de reducir la dimensión de la comunicación informal y mantener a los teletrabajadores presentes en la organización. Una buena idea es utilizar las mismas posibilidades que ofrecen las tecnologías de la comunicación e instalar servicios telemáticos de información, discusión e información.

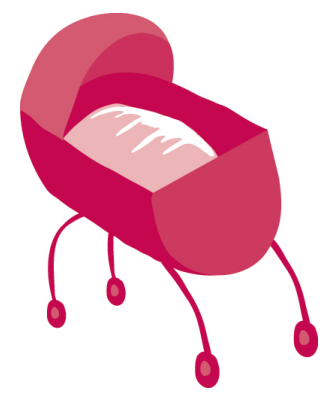


Cuando se implanta el teletrabajo se debe desarrollar e incrementar el espíritu de trabajo en equipo. La ausencia de comunicación puede disminuir la moral y la eficacia de los equipos. Los éxitos, los fracasos y la información clave deben compartirse para unir y motivar a los profesionales que trabajan en el mercado con objetivos comunes. Ello implica que la comunicación física debe mantenerse con el fin de no romper las relaciones de equipo (VIDEGAIN MURO, 1995, p. 399).

El año sabático es otra de las opciones de la flexibilidad que ofrecen algunas organizaciones. Eso sí, aquellos empleados que se acogen a este programa no perciben el cien por cien de su salario. Se trata de programas en los que el empleado se adhiere de manera voluntaria y deben estar aprobados por la dirección. Pueden contemplar la posibilidad de que el empleado trabaje para otras empresas, siempre que no sean competencia de la propia. Cuando el empleado regresa (puede volver antes de lo estipulado, así como puede acogerse a prórrogas pactadas con la empresa), mantiene su categoría profesional, tipo de contrato y salario.

Otras iniciativas que también conforman un entorno conciliador de la vida profesional y laboral son ayudas a gastos de enseñanza, subvenciones para compra de equipo informático, servicio médico, planes de pensiones...

Implantar una política de flexibilidad no siempre es posible, ya que no todos los puestos de trabajo tienen las mismas características. En general, las políticas relacionadas con el teletrabajo han mostrado ser muy eficaces sobre todo en las actividades comerciales y en los puestos de trabajo de servicios a clientes, donde dominan los horarios establecidos.

En determinados sectores, por ejemplo el informático, muchos proyectos implican que los empleados estén durante días trabajando de manera continua en dependencias de clientes. En esos casos, aplicar de manera arbitraria la flexibilidad desencadenaría una crisis en los proyectos por tener que regresar cada día a la sede central a presentar informes.

El desarrollo de tecnologías móviles ha sido muy eficaz para hacer frente este problema, pero no debemos apartar a estos trabajadores de la empresa, y siempre debe existir un espacio en el que puedan trabajar para cuando necesiten hacerlo desde la oficina.

En el caso de los directivos y mandos intermedios, esta flexibilidad puede adoptar una fórmula mixta. Los directivos tienen agendas apretadas que exigen presencia física en la oficina o en otro lugar para cumplir con sus compromisos. Pero también cuentan con herramientas que les permiten trabajar desde otros lugares.

Las máximas limitaciones a la hora de implantar políticas de flexibilidad están en la cultura y la mentalidad empresariales. Hay que despojarse de la idea de que se trabaja menos si se tiene jornada flexible o si se teletrabaja. 
Es posible que sea difícil llevar a cabo la flexibilidad en puestos secretariales o en trabajos por turnos. Entonces, la solución es crear puestos compartidos, esto es, asignar personas a funciones y no a puestos concretos, o crear equipos de trabajo polivalentes en los que varios empleados puedan realizar la misma tarea. El ejemplo de Sony es revelador: exige un horario mínimo y el resto es de libre elección (los empleados pueden incluso cambiar por vacaciones las tardes de los viernes) (CHINCHILLA et al., 2003). Las excedencias por maternidad son situaciones claras de cuándo aplicar la flexibilidad. En Airtel (ahora Vodafone), hay hombres que se han acogido a periodos de baja por paternidad.

Se aplica el apoyo profesional, que consiste en dar asesoría y formación al empleado para equilibrar su vida familiar y profesional. Para ello, las empresas incluyen asesoría psicológica, adaptación al trabajo o cursos de gestión del estrés. Las políticas de servicios tienen como objetivo reducir la carga de trabajo extra laboral del empleado. Para ello, se crean guarderías, servicios de limpieza, compensación económica para baby sitters que tienen que cubrir las horas de ausencia por trabajo...

Las prácticas más frecuentes son la flexibilidad de horarios y permisos de excedencia y por los beneficios extrajurídicos. Menos, por los apoyos profesionales, y casi nunca por servicios como guarderías. Profundizando en las prácticas más utilizadas, éstas son: el permiso de días libres, el coche de empresa para uso personal, el permiso de excedencia para el cuidado de niños, el trabajo a tiempo parcial y el asesoramiento de carrera profesional.

En un segundo nivel de prácticas se pueden citar: videoconferencia, asesoramiento financiero, horas de trabajo reducidas, seguros para hijos y cursos de gestión de conflictos (FLORES, 2005).

En general, los directores de recursos humanos suelen subestimar el grado de responsabilidad familiar de la cultura de empresa, mientras que los empleados suelen infraestimar la distribución de políticas familiarmente responsables en la empresa. Esto puede ser debido a una falta de comunicación de estas políticas al conjunto de la empresa, a la aplicación de las mismas sólo a niveles intermedios o a las dudas que les surgen a los empleados al utilizar algunas de estas políticas y correr el riesgo de ser señalizados con el dedo por disfrutar de privilegios que otros no tienen.

\section{Beneficiarios de la flexibilidad}

Los beneficiarios directos de las políticas de flexibilidad son la propia organización, los clientes y los empleados (IBM España, 2005). Además, podemos matizar sobre este último grupo que los beneficios sobre la mujer trabajadora son mayores, ya que sobre ellas recae la mayor parte de las responsabilidades domésticas. 
La retribución, aunque es importante, ya no es el único elemento que valoran los candidatos a la hora de elegir un empleo. Si bien es cierto que el salario debe ser competitivo en el mercado laboral, cada vez más, los elementos adicionales como beneficios sociales, posibilidades de desarrollo profesional, y sobre todo, la posibilidad de alcanzar un nivel suficiente de calidad de vida, se están convirtiendo en claves a la hora de escoger una empresa u otra. Aquellas empresas que así lo interpretan presentan un índice de rotatividad menor.

Otra de las ventajas es la motivación, ya que trabajar para empresas que muestran sensibilidad hacia circunstancias individuales mejora el compromiso de los empleados. Asimismo, la productividad se ve reforzada por el hecho de contar con profesionales más dispuestos a asumir responsabilidades y realizar actividades fuera de los horarios y oficina habituales. En el caso del teletrabajo se presenta la ventaja adicional de ahorro de espacio en la oficina, al implantarse las políticas de mesas compartidas (la más generalizada es la de tres empleados por mesa).

El beneficio principal para los clientes es la disponibilidad, porque las empresas tienen que actuar de manera mucho más rápida y flexible ante los cambios del entorno. La proliferación de la tecnología en las empresas ha permitido que puedan atenderse las necesidades de los clientes de manera casi instantánea. Éstos buscan proveedores con quienes puedan contar en cualquier circunstancia. Lógicamente, tener políticas de flexibilidad en las plantillas facilita la prestación de servicios a clientes de una manera flexible, y debe compensarse a los empleados que realizan un esfuerzo en horas fuera de la jornada laboral tradicional, de manera que puedan disponer de su tiempo y organizar su trabajo.

De manera indiscutible la flexibilidad de horario y de lugar de trabajo repercute de manera directa en la calidad de vida de los empleados y de sus familias. Si en una pirámide colocamos los tres niveles de felicidad (superficial, organizativa y personal), encontramos que en la cúspide, como la más valorada, está la felicidad personal, relativa a los grados de libertad. En un segundo nivel está la felicidad organizativa, que se refiere al ambiente abierto, equilibrado, las oportunidades y retos, el sueldo y cómo el empleado es valorado y aprobado ámbito cercano de trabajo. Ya en el último nivel, en la base, está la felicidad superficial, basada en la sucesión de momentos de celebración, generadores de un ambiente laboral motivador. Por ello, todas las ofertas que la empresa realice a los empleados para enriquecer esa vida personal serán muy valoradas en la permanencia y compromiso del trabajador (Factor H, 2003).

Un beneficio añadido es el incremento de la responsabilidad personal. Así, el empleado se convierte en el único responsable de su tiempo, de su trabajo, de su rendimiento y del desarrollo de su carrera profesional. Logra realizarse como trabajador y sentirse más a gusto en el desempeño de sus funciones. 
EL EQUILIBRIO PERSONAL Y PROFESIONAL COMO VALOR DE LA CULTURA CORPORATIVA • AMAIA ARRIBAS URRUTIA

\section{Otras experiencias}

El desarrollo de las políticas de flexibilidad no es una moda pasajera. Desde Europa nos llegan referencias de medidas que funcionan y a las que se acogen tanto los hombres como las mujeres. Hablar de flexibilidad es hablar de tiempo para el ocio, el deporte, la formación o la familia.

Ya en una conferencia de ministros europeos encargados de asuntos familiares, realizada en el 2001 (Ministerio de Trabajo y Asuntos Sociales de España, 2002) se examinaron las distintas políticas y mecanismos existentes en los estados europeos relativos a los trabajadores con responsabilidades familiares. Sus políticas tienen como principales objetivos flexibilizar los horarios de trabajo; ayudar a familias con hijos menores y con personas dependientes, y repartir equitativamente las responsabilidades entre el hombre y la mujer.

En general, las medidas implantadas responden a la necesidad social de cada país, por lo que son cuestiones culturales. Así, en los países escandinavos, las políticas persiguen facilitar a las mujeres trabajar y tener hijos; en Francia, aumentar la natalidad; en Gran Bretaña y Alemania, aliviar el impacto de la baja natalidad en la financiación de los planes de pensiones y los gastos sanitarios.

En función de estas necesidades sociales la reducción de la jornada es una política habitual en países como Chipre, Grecia, Noruega y Eslovenia, mientras que el trabajo a tiempo parcial o la elección de horarios son más habituales en Alemania, Francia, Grecia, España... Por ejemplo, Alemania e Irlanda han implantado medidas que favorecen el teletrabajo, y en Grecia existe la posibilidad de prolongar la jornada laboral durante seis meses al año a cambio de poderla reducir los seis siguientes (MACINNES, 2005).

Una medida creativa y apoyada por el gobierno belga es el crédito de tiempo, en el que el trabajador puede interrumpir momentáneamente su actividad profesional para dedicar un tiempo determinado a asuntos personales o familiares.

Para saber cómo evalúan las empresas la flexibilidad se pueden formular las siguientes preguntas en torno a tres ejes temáticos (políticas, programas y cultura):

1. ¿Ofrece la empresa flexibilidad en la jornada laboral?

2. ¿Es la empresa flexible al otorgar bajas que permitan atender las demandas familiares?

3. ¿Respeta la compañía la igualdad de oportunidades entre hombres y mujeres?

4. ¿Ofrece la organización información sobre el cuidado de niños y ancianos?

5. ¿Ha desarrollado un programa formal de armonización trabajo-familia?

6. ¿Ha comunicado formalmente la empresa dicho programa a través de los medios de comunicación internos? 
7. ¿Valora a sus empleados en función del cumplimiento de objetivos o de presencia física?

8. ¿Evita fomentar una cultura de adicción al trabajo o por el contrario se promociona a quien trabaja horas en exceso?

9. ¿Están los mandos intermedios sensibilizados para apoyar a los empleados cuando tienen que hacerse cargo de responsabilidades familiares?

10. Si el trabajador acepta más responsabilidad, ¿son capaces los directivos de discernir si ese sobreesfuerzo afectará a su equilibrio familia-trabajo?

\section{Conclusiones}

La conciliación de la vida personal (independientemente del modelo familiar y de convivencia) y laboral afecta a toda la sociedad. Involucra a todas las personas y a todas las edades, directa o indirectamente. La conciliación tiene un impacto directo sobre el ámbito del equipo humano (interno) y la sociedad y, por lo tanto, se trata de un eje trasversal de actuación para las distintas políticas públicas y/o empresariales.

El bienestar social pasa por un replanteamiento de los valores sociales y económicos, que permitan a las empresas optimizar su eficiencia mejorando la calidad de vida de las personas. Conciliar significa componer y ajustar las necesidades personales y las laborales sin que una exceda a la otra y mantenerlas proporcionalmente iguales. Este equilibrio no es estático, sino que va cambiando en función de las etapas laborales y las distintas situaciones personales.

Hay que superar dudas y prejuicios. Primero, hay que desechar la idea de que teletrabajar es una tarea que se ejerce siempre desde casa (existen telecentros en la mayoría de los hoteles).

Segundo, la flexibilidad de horarios no es un beneficio exclusivo de las mujeres trabajadoras. La preocupación por alcanzar un equilibrio entre la vida profesional y personal es común a ambos sexos.

Tercero, aquellos que consiguen un mejor equilibrio están más predispuestos a hacer un esfuerzo extraordinario cuando se necesita.

Cuarto, le flexibilidad no deteriora el trabajo individual ni el del departamento. Cada empleado es un caso diferente por lo que normalmente no todos ellos solicitan tener condiciones idénticas.

Quinto, es más importante preocuparse por el rendimiento y los resultados del empleado que por la presencia. 
Sexto, los empleados con buen historial de rendimiento suelen ser buenos candidatos a adoptar opciones de flexibilidad. Por otra parte, los resultados de muchos otros empleados pueden mejorar con un horario que les permita equilibrar mejor sus responsabilidades profesionales y personales.

Séptimo, el entorno empresarial y las actividades que se realizan en cada departamento conforman un escenario en continua transformación. Lo que no es posible en un momento determinado puede ser factible más adelante. Se trata de reconsiderar cada cierto tiempo las posibilidades de manera conjunta entre empleados y directivos.

Octavo, mantener un espíritu de equipo es difícil cuando los empleados no se encuentran ubicados en el mismo sitio, por lo que se exige un esfuerzo de planificación y de comunicación. Además, hay que aprovechar al máximo todas las oportunidades en las que haya contacto personal para mejorar este aspecto. La tecnología ofrece muchas soluciones (videoconferencias, chat en Internet, herramientas de trabajo colaborativas...) que faciliten la coordinación de equipos de personas que trabajan en diferentes sitios y que deban mantener el contacto para realizar sus tareas.

Noveno, en el caso de que un empleado no alcance los resultados esperados y ello esté directamente relacionado con la flexibilidad de que disfruta, evidentemente esas condiciones deben ser revisadas y modificadas.

Para qué sirve generar políticas para favorecer la flexibilidad de horarios en las empresas si no se actúa sobre la movilidad territorial o los horarios de los propios servicios públicos. Para qué sirve asegurar que una empresa gestiona a la perfección su impacto medioambiental si no es capaz de asegurar que se respeten los derechos humanos de todos sus trabajadores/as. Las políticas no deben limitarse a momentos concretos, sino que el compromiso que requiere la responsabilidad social tiene que traducirse en soluciones sostenibles independientemente del período temporal.

\section{Referencias}

ÁLVAREZ, Francisco. Trabajo y vida familiar: ¿Cómo conciliarlos? 2006. Disponible en: <http://www.monster.es>. Fecha de consulta: 16 de enero de 2007.

ARRIBAS URRUTIA, Amaia. Teletrabajo y comunicación interna: ¿incompatibilidad? Biblioteca On-line de Ciencias da Comunicação (Bocc), Universidad da Beira Interior: Portugal, marzo 2000. Disponible en: <http:// www.bocc.ubi.pt/pag/urrutia-amaia-teletrabajo.pdf $>$.

. Las nuevas tecnologías para la comunicación interna en la empresa. Tesis Doctoral. España: Ed. Universidad del País Vasco, 2002.

ASTELARRA, Judith. Políticas de género en la Unión Europea y algunos apuntes sobre América Latina. Publicaciones de Naciones Unidas. Santiago de Chile, 2004. 
EL EQUILIBRIO PERSONAL Y PROFESIONAL COMO VALOR DE LA CULTURA CORPORATIVA • AMAIA ARRIBAS URRUTIA

BAGUER ALCALÁ, Angel. !Alerta! Descubre de forma sencilla y práctica los problemas graves de tu empresa, sus vías de agua. Madrid: Ed. Díaz de Santos, 2005.

CALDERA MEJÍA, Rafael. Planeación estratégica de recursos humanos: conceptos y teoría. 2006. Texto completo disponible en: <www.eumed.net/libros/2006c/219/> (Edición electrónica gratuita).

CHINCHILLA, N.; POELMANS, S.; LEON, C. Políticas de conciliación trabajo-familia en 150 empresas españolas. Documento de investigación de IESE Business School. España: Universidad de Navarra, 2003.

ENCABO BALBÍN, Ana. La responsabilidad social de las empresas y los nuevos desafíos de la gestión empresarial. Espana: Universidad de Valencia, 2005.

FLORES, Patricia. Equilibrio vida profesional y personal: una responsabilidad de la Administración, la empresa y la sociedad. Capital Humano, n. 187, p. 60-64, abril 2005.

GARCÍA JIMÉNEZ, Jesús. La comunicación interna. Madrid: Ed. Díaz de Santos, 1998.

JIMÉNEZ, Alfonso; PIMENTEL, Manuel; ECHEVERRÍA, Mentxu. España 2010. Mercado laboral: proyecciones e implicaciones empresariales. Madrid: Ed. Díaz de Santos, 2002.

La conciliación según IBM o cómo ser flexibles sin romperse. Capital Humano. IBM España, n. 187, p. 40-58, abril 2005.

Las claves para un entorno de trabajo ideal. Factor H, n. 6, p. 4-6, marzo-abril 2003.

MACINNES, J. Diez mitos sobre la conciliación de la vida laboral y familiar. Cuadernos de Relaciones Laborales. Universidad Complutense de Madrid, n. 23, p. 35-71, 2005.

REVISTA DEL MINISTERIO DE TRABAJO Y ASUNTOS SOCIALES. España: 2002. (n. 40)

LORETO Marchant R. (ed.) Actualizaciones para el management y el desarrollo organizativo. 2007. Texto completo disponible en: <www.eumed.net/libros/2007a/223/> (Edición electrónica gratuita).

PÉREZ PÉREZ, Manuela; DE LUIS, Pilar; MARTINEZ SÁNCHEZ, Angel. La adopción y difusión potencial del teletrabajo: estudio de factores explicativos basados en teorías organizacionales. Ponencia presentada en el XI Congreso Nacional de ACEDE. España: Universidad de Zaragoza, 2001.

PUCHOL, Luis et al. Nuevos casos en dirección y gestión de recursos humanos. Madrid: Ed. Díaz de Santos, 2005.

RIMBAU, Eva y TRIADÓ, Xavier. La flexibilidad en la empresa. Capital Humano, n.197, p. 38-48, 2006.

QUINTANILLA NAVARRO, Beatriz. La conciliación de la vida laboral y familiar en el marco jurídico actual. Cuadernos de Relaciones Laborales, n. 23, p. 95-129, 2005.

SERVICIO NAVARRO DE EMPLEO, CEIN, ANEL, Cederna-Garalur. Servicios avanzados a empresas de mejora de competitividad. Informe. España, 2004.

VIDEGAIN MURO, Juan. IBM España, una experiencia europea de teletrabajo. Alta Dirección, n.184, p. 395401, nov.-dic. 1995. 